DEPARTMENT OF THE IITERIOR

U.S. GEOLOGICAL SURVEY

\title{
1DT - an interactive, screen-oriented microcomputer program for simulation of 1-dimensional geothermal histories
}

by

Ralph A. Haugerud ${ }^{1}$

U.S. Geological Survey / National Research Council

Open-File Report $86-511$

This report is preliminary and has not been reviewed for conformity with U.S. Geological survey editorial standards. Any use of trade names is for descriptive purposes only and loes not imply endorsement by the USGS.

$1_{959}$ National Center

Reston, Virginia 22092 


\section{Abstract}

IDT is an interactive Pascal program for one-dimensional modelling of conductive and advective heat transfer in a geologic context. The program allows for user-specified physical properties, basal boundary conditions, internal heat generation, tectonic deformation, and rates of erosion. Input is from the keyboard; output is in the form of several graphic displays which may be printed during program execution, as well as a disk file of the temperature-depth conditions experienced by specified material points during the evolution of the model. IDT runs on the IBM PC and compatible microcomputers and should be easily portable to many other machines.

\section{Introduction}

Several recent studies (e.g. Draper and Bone, 1981; England and Thompson, 1984; Chamberlain and England, 1985) have exploited the power of finite difference solutions to the l-dimensional heat-transfer equation. These authors have tapped a vein of rich ore that has not yet been exhausted, and reading about thermal models is no substitute for first-hand experience in gaining an appreciation for the phenomena of geologic heat transfer and the limitations of numerical modelling. This report describes IDT, a relatively portable, easy-to-use, microcomputer program for thermal modelling that allows for a large range of input parameters. It is hoped that others will find this program useful for teaching, self-education, and research.

\section{To Install 1DT}

The program disk has source and object code for IBM PC-compatible microcomputers with appropriate graphics hardware, a routine to customize the object code for different terminals, and a batch file, INSTALL.BAT, to install IDT. This batch file erases the source code files, the terminal customization routine, and object code for alternate graphics hardware. Make a copy of the disk before you do anything else! Files on the program disk are detailed in Appendix 1.

To install 1DT,

- Put a write-protect tab on the disk

- Copy the disk

- Log onto the drive the disk is in

- For machines with Hercules graphics boards, enter INSTALI HGC. For other graphics hardware, enter INSTALL IBM. INSTALL will rename some files, customize the programs for monitor characteristics (you will have to answer the same question about monitor characteristics three times) and then ERASE the installation programs and the object code for other graphics hardware.

You can now run ID'T. Answer "?" at the first question. Though further explanation is offered below, the program is intended to be self-explanatory. 
Description of Program 1DT

For one-dimensional conductive and advective transfer of heat, the governing equation is

$$
\frac{\delta T}{--}=\frac{\delta^{2} T}{\delta z^{2}}-\frac{\delta T}{\delta z}+\frac{A}{\phi C}
$$

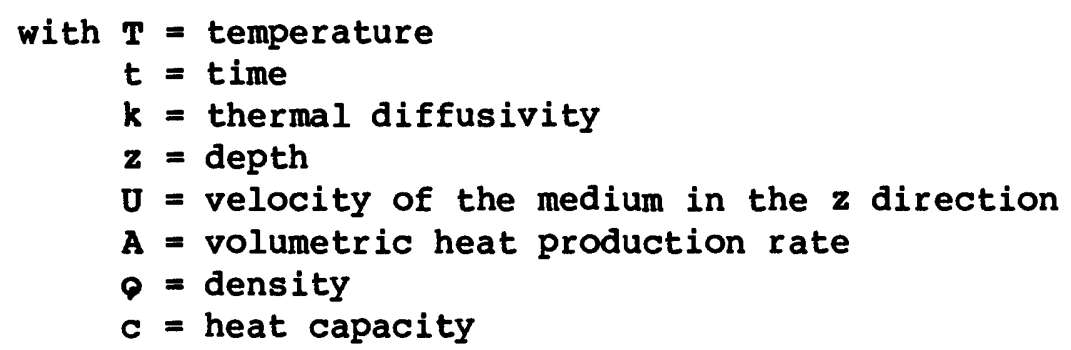

For finite simulations it is necessary to specify initial conditions and boundary conditions. A program to simulate conductive and advective heat transfer thus needs to provide the following:

an algorithm to solve equation (1)

an input procedure

physical properties

a heat generation function $A$

a velocity function $U$

boundary conditions

an initial temperature distribution

an output procedure

These aspects of IDT are described below.

Algorithm to Solve Equation (1) Equation (1) is solved by the Crank-Nicolson finite difference technique (Appendix 2). In this technique, the partial derivatives with respect to time are approximated by the average of the value at time = present and the value at time = present + timestep. The space derivatives are replaced by the average of their values at time $=$ present and at time $=$ present + timestep, with the space derivatives approximated by considering the change in values over the interval $\mathrm{z}$ - spacestep to $\mathrm{Z}+$ spacestep.

Inherent theoretical limits to the accuracy of finite-difference techniques are discussed by Smith (1985) and Carnahan and others (1969). Accuracy is a function of time- and space-step size. Possible ill effects due to steps that are too large can be tested empirically by running a model with a smaller steps--all that is needed is enough machine memory and more time. Several authors have tested accuracy by modelling simple scenarios for which analytic solutions are available (e.g. Draper and Bone, 1981; Haugerud, 1985). Results of these tests are encouraging, with errors in most cases no greater than a few percent.

Explicit finite difference techniques have very severe limits on permissible relative step sizes. For pure conductive transport, if the ratio

$$
r=\text { diffusivity * timestep / spacestep }{ }^{2}
$$


exceeds 0.5 the explicit calculation is numerically unstable. The effect is not the least bit subtle. With a program that plots the geotherm every time-step, one can watch temperatures at a given gridpoint oscillate with ever-increasing amplitude until the machine shuts down due to floating-point overflow.

The implicit Crank-Nicolson technique used by IDT is unconditional$1 y$ stable. However, if $r$ is large $(>>1)$ and if there are local extreme curvatures in the geothermal gradient, temperatures in the vicinity of the extreme curvature will oscillate. The oscillations decay with time and do not appear to damage accuracy outside the affected region. Geothermal behavior within the affected region is manifestly not correctly depicted. Small time-steps are thus required to examine thermal histories in the spatial and temporal vicinity of simulated faults. Lengthy run-times can be avoided by changing to a longer time-step after local extreme curvatures in the gradient have decayed. Thermal discontinuities at external boundaries can also strain the Crank-Nicolson technique; these limitations are outlined by Smith (1985).

Input The first screen of IDT prompts the user to read a sample input file of model parameters, or for the name of an alternate input file. This file is read, then the parameters are presented screen by screen with options to accept (or change) each screen. After all screens have been approved, the user is then asked to accept (or change) the input parameters as a whole.

After a model is run, the user is offered the option of saving the modified parameters to a disk file for re-use or further editing at another session. The input parameters may also be printed at this time (Figure 1).

Note that one always modifies a pre-existing model rather than creating a model out of whole cloth.

Physical Properties Thermal conductivity, density and heat capacity are considered constant throughout the modelled space. The user is asked to specify these values.

It would be fairly easy to modify the code to allow for place-toplace variation of density and heat capacity, though this would make conversion of depth to pressure more complicated. It is possible to allow for place-to-place variation in thermal conductivity but this would require substantial re-writing of the procedure that implements the Crank-Nicolson technique.

Heat Generation Internal heat generation is specified as a slab/ sawtooth function, with values interpolated between the values at the boundaries of specified layers. The user is asked to specify the number of layers, original depth to layer boundaries and the heat production at the top and base of each layer. Heat production below the base of the last layer is assumed to be zero. As the model evolves, through burial, uplift, and internal deformation, the positions of the layer boundaries are moved appropriately and the heat generation at any point is defined by linear interpolation from the values at the layer boundaries.

The top of the first layer (at the surface) does not move with time, though the base of the layer is moved. Consequently, heat generation in the uppermost layer of a model with erosion or uplift is depicted correctly only if the uppermost layer is a slab with internal heat generation constant with depth. IDT is not capable of repeating 
(or excising) layers in the heat-generation structure during "thrust" events (see below). The program thus cannot easily duplicate the "thrust" scenario of England and Thompson (1984), in which a $35 \mathrm{~km}$ thick crust with a $10 \mathrm{~km}$ thick radioactive upper layer is repeated by thrusting. Attempting to create such a model with 1DT will lead to an afterthrusting crust with a $45 \mathrm{~km}$ thick upper radioactive layer. However, such a thrust scenario can be duplicated by using the prescribed initial gradient feature and setting the initial thermal profile and heat generation structure to their post-thrusting configuration.

Velocity The model frame of reference is the surface, $z=0$, with $z$ increasing downwards. Negative velocities then correspond to uplift, positive velocities to burial. The velocity function used is

$$
u=a+b z
$$

with $a$ and $b$ constant. $a$ is the rate of en-bloc translation, whereas non-zero values of $b$ produce homogeneous strain. Different velocities may be prescribed for several stages in a burial/uplift history, with stages described by the time at the end of each stage.

In addition to advective heat transfer described by the velocity term, 1DT incorporates the option for an instantaneous "thrust" (cf. England and Thompson, 1984) at the end of each uplift stage. In a thrust event, temperatures below a certain level (the thrust sheet thickness) are assigned values corresponding to those a certain distance (the amount of section duplicated) above this level (Figure 2). Temperature at the mesh-point closest to the thrust is then set to the average of the values at the mesh-points above and below. Low-angle normal faults may be simulated by specifying a "thrust" that duplicates a negative amount of section. Thrust sheet boundaries need not correspond to heat-generation layer boundaries.

The depth-time plot (see Output, below) is useful for insuring that the velocity function specified is the one intended.

Boundary Conditions The surface temperature is set at 0 degrees in all cases. Basal temperatures and gradients are calculated at the beginning of a model run. One has the option of then keeping the basal flux (= gradient * conductivity) constant or keeping the basal temperature constant.

Initial Temperature Distribution Initial temperatures are calculated equilibrium values for the specified heat generation, surface temperature and basal flux, or may be prescribed. If one chooses to prescribe the initial temperature, there are two options: import a geotherm (and initial time) from a disk file created by using the File option during an earlier run; and prescribe a geotherm by specifying temperatures at arbitrary increasing depths. In either case one has the option of editing the resulting temperatures.

Output IDT creates five pieces of output. During program execution, a graph of depth vs. temperature is displayed (Figure 2). The depth-T paths followed by specified material points and the geotherm are drawn on this graph at specified time intervals. At the same time, records of depth-T values for these same points are written to a disk file. After the finite difference calculation is completed, the user has the option of re-opening this disk file and using it to construct graphs of depth-time (figure 3), temperature-time (figure 4) and depthtemperature (figure 5) paths. 
Hard copy of the various graphs can be created using the screenprint option displayed on various menus within the program. This option invokes a TurboGraphix procedure that supports series MX, RX, and FX Epson printers only. To produce hard copy on other printers will require using a graphics screen-dump utility that is external to program IDT or modifying IDT. Hardcopy will be automatically created if one specifies the automatic printout option on the OUTPUT screen.

The number and initial depths of the points for which P-T histories are tracked is specified in the OUTPUT screen. Negative initial depths can be specified, to track paths of points yet to be created by thrusting or subsidence and deposition.

Parameters specified in the OUTPUT screen are not saved to a disk file with the model parameters; rather, they are initialized (in procedure Header) every time the program is run. Different default values for the output procedures can be set by changing the appropriate statements at the beginning of this procedure.

After exiting program IDT one may list the records of depth-T values using program IISTPTT. LISTPTT can write this information to the screen, a disk file, or the printer.

Screens saved using the Savescreen option may be viewed and printed with program VIEW.

Help

In the first screen the user is offered the option of viewing a file of helpful hints that in part repeats and and in part complements this discussion.

At almost all points in IDT typing $\langle E S C\rangle$, or if nothing happens, control-C will either abort the program or lead to an option to abort the program.

While 'thinking', program execution may be suspended by typing 〈ESC. This will branch to a menu that offers the options Abort, Change DT, Printscreen, Savescreen, New problem, File and Return. These options are invoked by typing the first letter of each.

Program Structure

The structure of the program is illustrated in Figure 6.

Implementation and Portability

IDT is written in TurboPascal, a dialect of Pascal published by Borland International. Graphic displays are implemented with the TurboGraphix package offered by Borland. The program disk has versions of IDT compiled on an IBM PC with a Hercules monochrome graphics card and an IBM PC with an IBM graphics card. IDT has been run on an IBM PC with the Hercules monochrome graphics board, an IBM PC with an IBM graphics board, a Toshiba TI100, and a Compaq. It should run on most IBM PCcompatible microcomputers with appropriate graphics hardware. 
Modifying the program

Users are encouraged to modify 1DT. This will require the TurboPascal compiler as well as the TurboGraphix Toolbox. These products are available for most 8088- and 8086-based machines. The compiler is also available for 780 -based machines. It should not be difficult to adapt IDT to a machine for which the TurboGraphix package is not available, provided a version of the compiler is available and the machine has dotaddressable screen graphics; indeed, IDT was written on such a machine. To do so will require writing versions of the graphics procedures described in Appendix 3. An example alternate graphics package is given in the file IDTIA.PAS on the program disk.

Likely modifications include re-dimensioning various arrays in the program to allow for more stages in the model history, allow for more heat-generation layers, or fit the program into a machine with less RAM; changing the output routines; and modifying the velocity and heat generation functions. Note that if arrays within the record type MODEL are re-dimensioned, it will be necessary to run the program 'blind' to construct an input file. If this is done, set the boolean constant FirstTime (at the head of file lDT.PAS) to true, compile, run the program, save the model parameters to the file SAMPLE.IDT, set FirstTime to false, and recompile.

Modifying the velocity and heat generation functions will require substantial rewriting of the input routines. However, the CrankNicolson algorithm (procedure $\mathrm{CN}$ ) and the output routines should require little or no modification.

1DT.MSG is the text file read by the procedure invoked by "?" at the first question. This file may be modified with any text editor, using non-document mode.

\section{Error Handling}

There is little in the way of error trapping in 1DT. Io errors (character instead of numeric input, for example), disk errors and the like will be fatal. If by some chance a fatal error occurs while in graphics mode, the program will return to the operating system level while in graphics mode. Resetting may then be required.

Bugs

Pay attention to your results. If they appear unreasonable, they may bel There are undoubtedly a few bugs still lurking in the 2,000+ lines of code that constitute 1DT. If you do discover any problems, please contact the author. Complete documentation of the sequence of instructions that produce anomalous behavior will be very helpful. The sections of code that use the velocity function to prescribe heat generation in the evolving model are the weakest part of the program and the most likely source of failure. To examine detailed behavior of a model, and verify that heat generation and velocity are properly assigned, there is a Troubleshoot option available when program execution is suspended by typing 〈ESC) (see Help, above). This option is not on the menu. Typing 〈T〉 will allow one to examine the current time; surface temperature; basal flux; and temperatures, velocities, and 
heat generation at each grid point. The Troubleshoot option will erase large parts of the graphic display on most machines.

References Cited

Carnahan, B., Luther, H. A., and Wilkes, J. 0., 1969, Applied numerical methods: New York, John wiley and Sons, 604 p.

Chamberlain, C. P., and England, P. C., 1985, The Acadian thermal history of the Merrimack Synclinorium in New Hampshire: Journal of Geology, v. 93, p. 593-602.

Draper, G., and Bone, R., 1981, Denudation rates, thermal evolution, and preservation of blueschist terrains: Journal of Geology, v. 89, p. 602-613.

England, P. C., and Thompson, A. B., 1984, Pressure-temperature-time paths of regional metamorphism, Part $I$. Heat transfer during the evolution of regions of thickened continental crust: Journal of Petrology, v. 25, p. 894-928.

Haugerud, R. A., 1985, Geology of the Hozameen Group and the Ross Lake shear zone, Maselpanik area, North Cascades, southwest British Columbia (PhD dissertation): Seattle, University of Washington, 263 p.

Smith, G. D., 1985, Numerical solution of partial differential equations: finite difference methods (3rd edition): oxford, oxford University Press, 337 p. 
Appendix 1. A note on the program disk

The program disk is an MS-DOS format $51 / 4 "$ floppy disk with the following files:
IDTCOM. IBM
object code for PC with IBM graphics card
IDTCOM.HGC
object code for PC with Hercules graphics card One of these must be renamed IDT.COM.
IDT000.IBM overlay file for IDT with IBM graphics
IDT000.HGC overlay file for IDT with Hercules graphics
The appropriate file must be renamed IDT.000

IDT.COM, IDT.000, and the 6 files below must be present on the default drive/directory when running IDT.

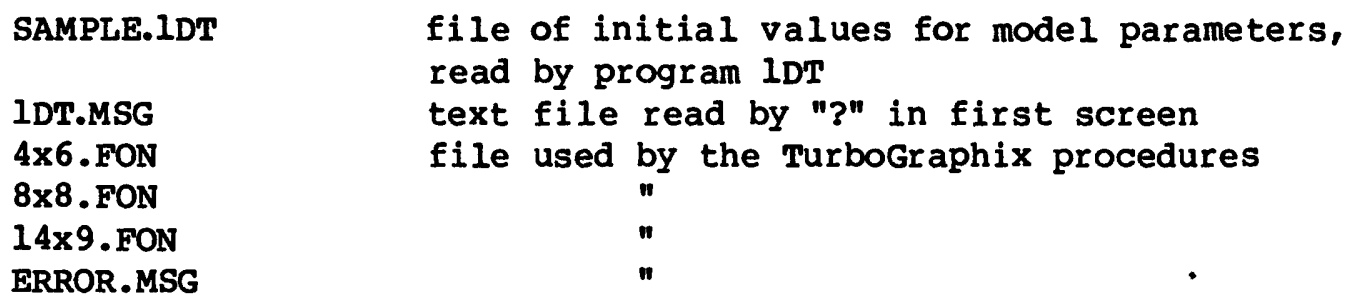

The following text files are source code for IDT and need not be present to run the program.

$\begin{array}{lclc}\text { IDT.PAS } & \text { main program } & \text { IDT5.PAS } & \text { include file for IDT } \\ \text { IDT1.PAS } & \text { include file for IDT } & \text { IDT6.PAS } & " \\ \text { IDT2.PAS } & \text { " } & \text { IDT7.PAS } & " \\ \text { IDT3.PAS } & " & \text { IDT8.PAS } & " \\ \text { IDT4.PAS } & " & \text { IDT9.PAS } & " \\ \text { IDT4A.PAS } & " & \end{array}$

IDTIA.PAS is an include file for IDT that contains a set of surrogate, non-IBM graphics routines. It is not included in the compiled versions of the program on this disk.

The 5 files below are source and object code for LISTPTT and VIEW, utilities for viewing the output files created by IDT and viewing and printing saved screen images.

LISTPTT. PAS

LISTPTT. COM
VIEW.PAS

VIEWCOM.IBM

VIEWCOM. HGC

IDTINST.COM is a terminal installation routine that uses the files IDTINST.MSG and IDTINST.DTA. INSTALL.BAT is a batch file that renames files; configures IDT, VIEW, and LISTPTT; invokes the program IDTINST.COM; and erases all files with the extension PAS, files with the extension HGC or IBM, and files with that start with the designation IDTINST. 
Appendix 2. The Crank-Nicolson method

One-dimensional heat transfer by conduction and advection is governed by the conservation equation

$$
\frac{\delta T}{\delta t}=\frac{\delta^{2} T}{\delta z^{2}}-\frac{\delta T}{\delta z}+\frac{A}{\phi C}
$$

with $T$ = temperature,

$t=$ time,

$k$ = thermal diffusivity,

$\mathbf{z}=$ depth,

$U=$ velocity of the medium in the $z$ direction

$A=$ volumetric heat production rate

$Q=$ density

$c=$ heat capacity

Consider discrete intervals of space $D z$ and time $D t$, such that $z=$ iDz and $t=n D t$. The partial derivatives $\delta^{2} \mathrm{~T} / 6 \mathrm{z}^{2}$ and $\mathrm{cr} / 6 \mathrm{z}$ can be replaced with their finite-difference equivalents \&zzT and \&zT, such that

$$
\& z z T=\frac{T_{i+1}+T_{i-1}-2 T_{i}}{D z} \quad \text { and } \& z T=\frac{T_{i+1}-T_{i-1}}{2 D^{2}}
$$

If we approximate $6 r / b t$ by $\left(T^{n+1}-T^{n}\right) / D t$, and replace the right-hand side of equation (1) with the average of its finite-difference equivalents at time $t=n D t$ and $t=(n+1) D t$, we have the Crank-Nicholson approximation:

$\underset{T^{n+1}-T^{n}}{D t}=\frac{-}{2}\left[\begin{array}{cc}\left(k \& z z T^{n+1}-U^{n+1} \& z T^{n+1}+A^{n+1}\right)+\left(k \& z Z T^{n}-U^{n} \& z T^{n}+A^{n}\right) \\ -\infty & \phi C\end{array}\right]$

Rearranging, collecting terms, and letting

$$
I=\frac{k D t}{2 D z^{2}} \quad V_{i}^{n}=-\frac{U^{n} D t}{4 D z} \quad \text { and } H_{i}^{n}=-\frac{A^{n} D t}{2 \phi c} \text {, }
$$

gives

$$
\begin{aligned}
\left(-L-V_{i}^{n+1}\right) T_{i-1}^{n+1}+(1+2 L) T_{i}^{n+1}+\left(-L+V_{i}^{n+1}\right) T_{i+1}^{n+1} \\
=\left(L+V_{i}^{n}\right) T_{i-1}^{n}+(1-2 L) T_{i}^{n}+\left(L-V_{i}^{n}\right) T_{i+1}^{n}+H_{i}^{n+1}+B_{i}^{n}
\end{aligned}
$$

for $0<i<i \max$.

This may be written 
$a_{i} T_{i-1}^{n+1}+b_{i} T_{i}^{n+1}+c_{i} T_{i+1}^{n+1}=d_{i}$

with

$$
\begin{aligned}
& a_{i}=-L+v_{i}^{n+1} \\
& b_{i}=1+2 L
\end{aligned}
$$$$
c_{i}=-L-V_{i}^{n+1}
$$$$
\text { and } d_{i}=\text { right-hand side of equation (3). }
$$

In a geological setting, it is useful to consider cases in which surface temperature, $T_{0}$, is a known function of time. In this case, for $i=1$, equation (4a) can be rewritten as

$$
\mathrm{b}_{1} \mathrm{~T}_{1}+\mathrm{c}_{1} \mathrm{~T}_{2}=\mathrm{d}_{1}
$$

with

$$
d_{1}=\left(L+V_{i}^{n}\right) T_{i-1}+(1-2 L) T_{i}^{n}+\left(L-V_{i}^{n}\right) T_{i+1}^{n}+H_{i}^{n+1}+H_{i}^{n}-\left(-L-V_{i}^{n+1}\right) T_{0}^{n+1}
$$

A known basal gradient, $6 T / b z$ at $z=i \max (D z)$, at $t$ ime $t=n D t$, can be replaced by its central difference approximation, BG, with

$$
\begin{aligned}
& B G^{n}=\left(T_{i \max +1}^{n}-T_{i \max -1}^{n}\right) /(2 \mathrm{Dz}) \\
& \text { Rearranging, } T_{i \max +1}^{\mathrm{n}}=\mathrm{T}_{\mathrm{imax}-1}^{\mathrm{n}}+2 \mathrm{Dz} \mathrm{BG}^{\mathrm{n}} \text { and equation (4a), for } \\
& i=\text { imax, becomes } \\
& a_{i \max } T_{i \max -1}+b_{i \max } T_{i \max }=a_{i \max }
\end{aligned}
$$

$a_{\text {imax }}=-2 \mathrm{~L}$ and

$$
d_{i m a x}=2 L T_{i-1}+2(1-L) T_{i}^{n}+H_{i}^{n+1}+H_{i}^{n}+2 D z\left(\left(L-V_{i}^{n}\right) B G^{n}-\left(-L+V_{i}^{n+1}\right) B G^{n+1}\right)
$$

A known basal temperature can be treated in the same fashion as a known surface temperatures.

Equations $4 a, 4 b$, and $4 c$ constitute a set of $m$ (=imax) simultaneous equations in $m$ unknowns, in which the unknowns are temperatures at each gridpoint at time $=(n+1) D t$. The equations are tridiagonal, that is, in the form

$$
\begin{aligned}
& b_{1} T_{1}+c_{1} T_{2} \\
& =d_{1} \\
& a_{2} T_{1}+b_{2} T_{2}+c_{2} T_{3} \\
& =d_{2} \\
& a_{3} T_{2}+b_{3} T_{3}+c_{3} T_{4} \\
& =d_{3} \\
& a_{m} T_{m-1}+b_{m} T_{m} \\
& =\mathrm{d}_{\mathrm{m}} \text {. }
\end{aligned}
$$


IDT uses these relations to predict temperatures in a onedimensional crust. The tridiagonal set of equations is solved by the procedure TRIDAG, translated from the FORTRAN subroutine presented by Carnahan and others $(1969$, p. 446).

Smith (1985) indicates that under certain conditions the Gaussian elimination algorithm implemented in TRIDAG is subjected to erroramplification. An option to check for susceptibility to machine error in TRIDAG is presented during the input portion of 1DT. Ordinarily this should not be selected. If the option is selected, values for $a, b$, and $c$ for $i-1, i$, and $i+1$ will be printed whenever the inequalities

$$
b_{i}>\operatorname{abs}\left(a_{i}+c_{i}\right) \text { and } b_{i}>\operatorname{abs}\left(c_{i-1}+a_{1+1}\right)
$$

with $a_{1}=c_{m}=0$, are not preserved. 
Appendix 3. Graphics procedures used by IDT

IDT uses graphics procedures provided by the TurboGraphix Toolbox marketed by Borland International. If IDT is implemented on a machine for which the TurboGraphix Toolbox is not available, surrogates for the procedures listed here must be written. A set of surrogates for use on the Otrona Attache, a CP/M machine with dot-addressable graphics, is included on the program disk as file IDTIA.PAS.

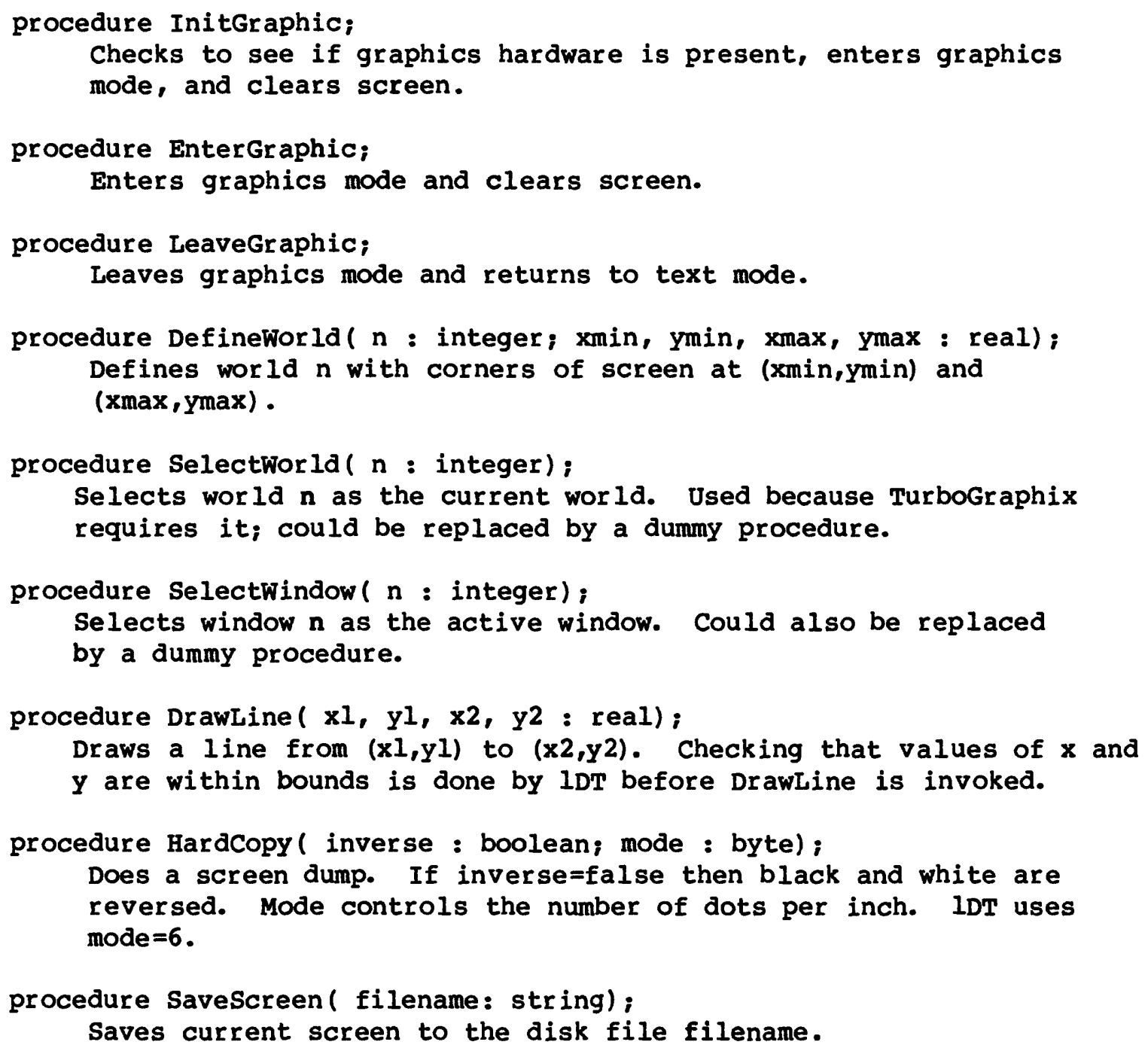




\section{Figure Captions}

Figure 1. Listing of model parameters produced by IDT. Model is named "sample" and is depicted in figures 2-5. The velocity function prescribed is for a 3-stage deformation history, with 0.5 million years of quiescence ended by a "thrust" event, another $10 \mathrm{million}$ years of quiescence, and then $20 \mathrm{million}$ years of erosion at $0.4 \mathrm{~mm} / \mathrm{yr}$ coupled with homogeneous thinning at a strain rate of $1 \times 10^{-15} \mathrm{sec}^{-1}$.

Figure 2. Initial output of 1DT. Vertical axes are depth in $\mathrm{km}$ with ticks at $10 \mathrm{~km}$ intervals; horizontal axes are temperature in degrees Celsius. Sweeping lines are instantaneous geotherms. A) Sample model immediately after "thrust" event. Note that "thrust" is at depth of 15 $\mathrm{km}$ and repeats $10 \mathrm{~km}$ of thermal section. Vertical and straight diagonal lines are P-T paths of discrete rock masses. B) After completion of model run (time $>50 \mathrm{my}$ ). Geotherms have been drawn at 2 my intervals.

Figure 3. Depth-time plot produced by 1DT. Vertical axis is depth in $\mathrm{km}$; horizontal axis is time in millions of years. Curves are paths followed by selected points. Note rapid burial by thrusting, quiescence, and then uplift/erosion (all points move to shallower depths) coupled with homogeneous thinning (all points move closer together).

Figure 4. Temperature-time plot produced by IDT. Vertical axis is temperature; horizontal axis is time in millions of years.

Figure 5. Depth-temperature (P-T) plot produced by 1DT. Vertical axis is depth in $\mathrm{km}$, horizontal axis is temperature in degrees Celsius.

Figure 6. Flow chart for use of program lDT. 
Sample

thermal conductivity $(W m-1 K-1)=2.50 E+00$

density $(\mathrm{kg} \mathrm{m}-3)=2.78 \mathrm{E}+03$ heat capacity $(\mathrm{J} \mathrm{K}-1 \mathrm{~kg}-1)=9.00 \mathrm{E}+02$

\# layers $=1 \quad$ \# stages in uplift history $=3$

total model time $($ years $)=5.000 E+07$

surface temperature $=0 C_{;}$initial basal flux $(\mathrm{W} m-2)=3.00 E-02$ constant basal flux

Heat gereration in the modelled space:

- surface-

LAYER 1 heat generation at top of layer $=2.00 \mathrm{E}-06 \mathrm{~W} \mathrm{~m}-3$

heat generation at base of layer $=2.00 \mathrm{E}-06 \mathrm{~W} \mathrm{m-3}$

heat generation $=0.0$

Initial surface flux $=0.06 \mathrm{~W} \mathrm{m-2}$

Equilibrium initial gradient

Velocities relative to the earth's surface (negative values for uplift): time at end of en-bloc velocity strain rate "thrust" event

Stage 1 stage, in yrs

Stage 2

5. $00 \mathrm{E}+05$ in $\mathrm{mm} / \mathrm{yr}$

$1.05 E+07$

0.00

in $\mathrm{sec}-1$

$0.00 E+00$

( $Y$ or $N$ )

Stage 3

$3.05 E+07$

0.00

$0.00 E+00$

$-1.0 E-15$

$Y$
$N$
$N$
time
thickness of years thrust sheet $(\mathrm{km})$
5. $00 \mathrm{E}+05$
15.0
amount of section
duplicated $(\mathrm{km})$
10.0

time-step $($ years $)=2.00 E+05$

$\#$ of depth steps $=100$ depth-step size $(\mathrm{km})=1.00$

model $\operatorname{depth}(\mathrm{km})=100.00$

P-T-t data recorded every 1 timesteps

10 timesteps between plots of the geotherm

P-T paths tracked for 6 points:

point $1 \quad$ initial depth $(\mathrm{km})=-5.00$

point 2 initial depth $(\mathrm{km})=0.00$

point 3 initial depth $(\mathrm{km})=5.00$

point 4 initial depth $(\mathrm{km})=10.00$

point $5 \quad$ initial depth $(\mathrm{km})=15.00$

point 6 initial depth $(\mathrm{km})=20.00$

P-T-t records saved to file Sample.PTt

Figure 1. 


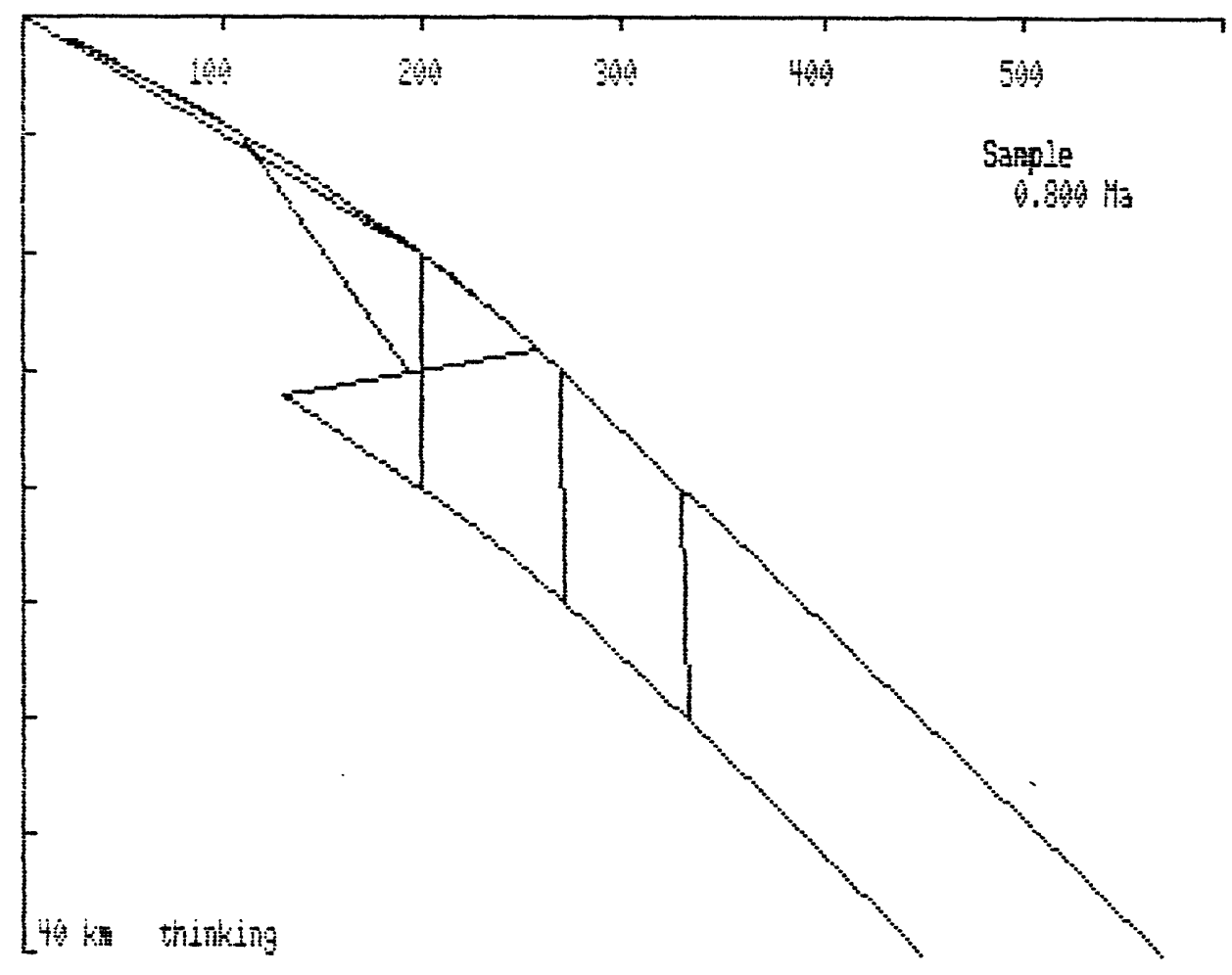

Figure 2 .

(A)

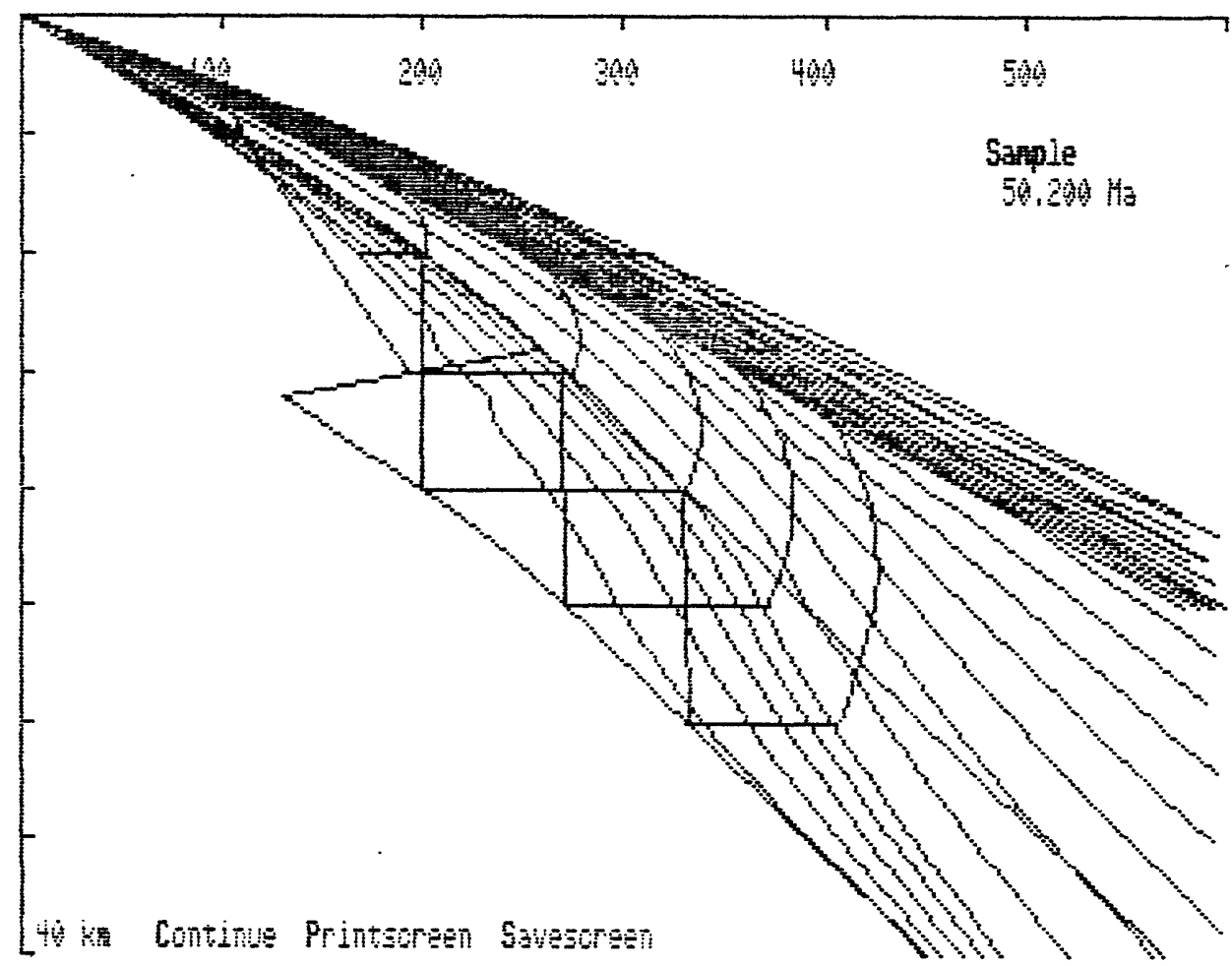

(B) 


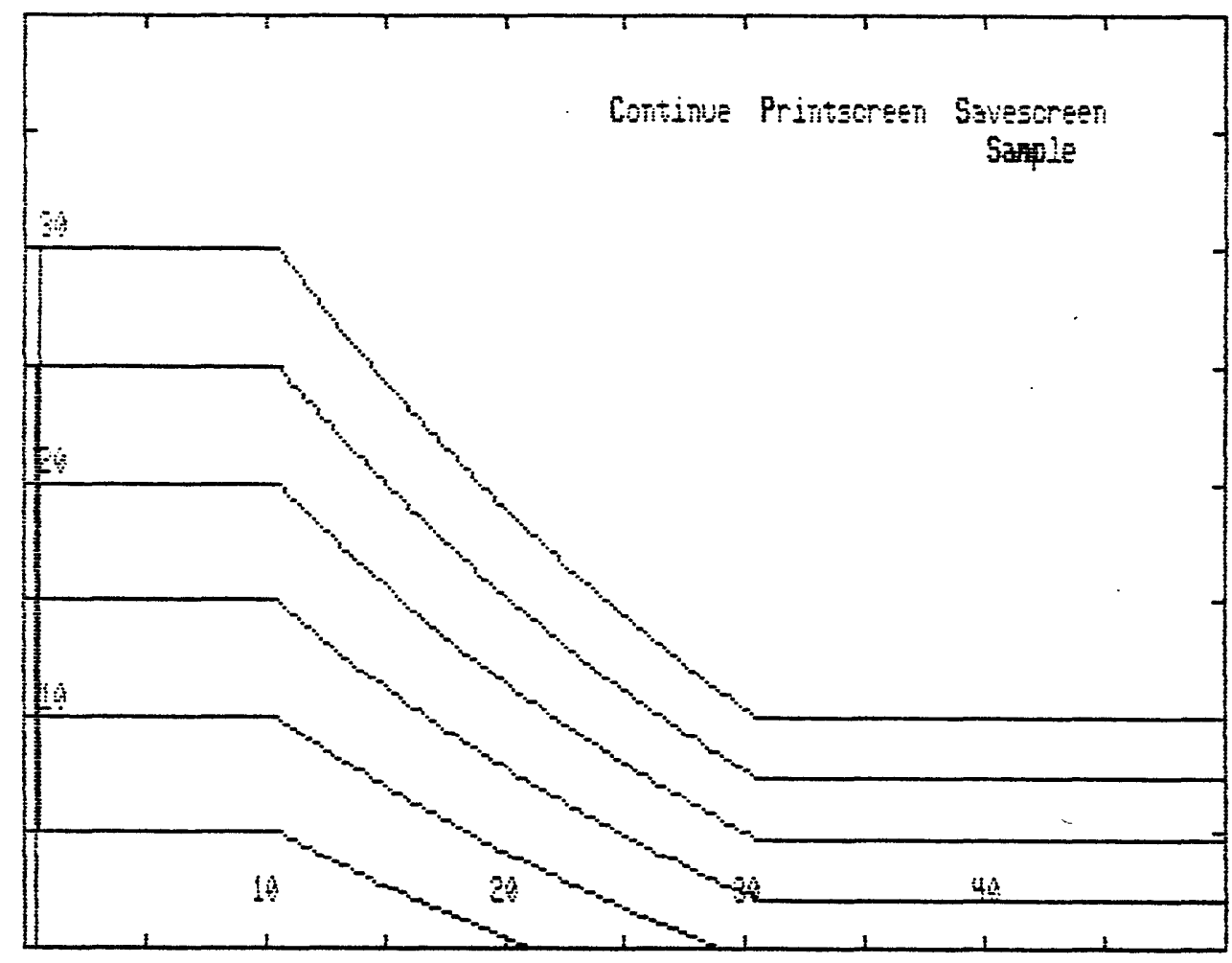

Figure 3.

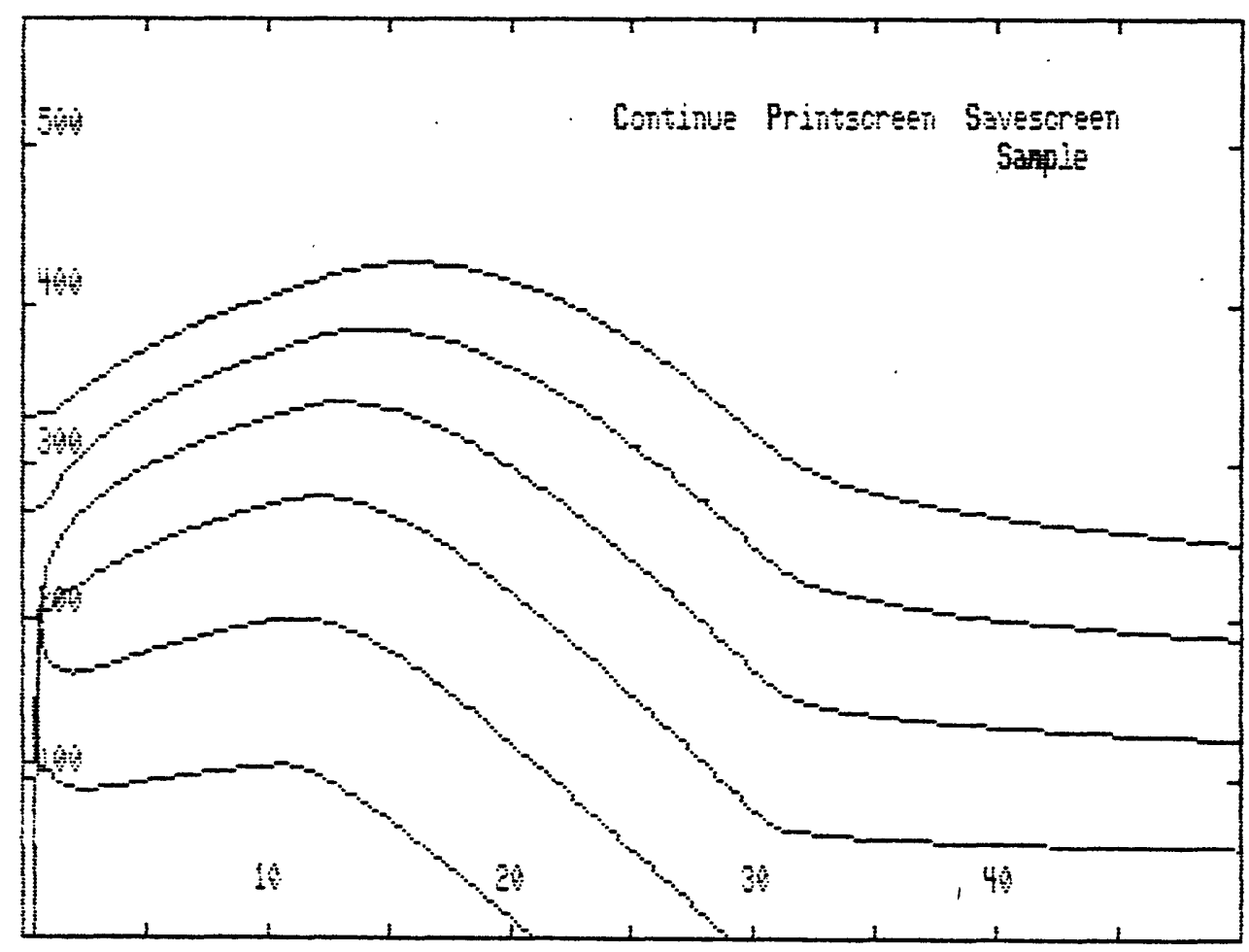

Figure 4. 


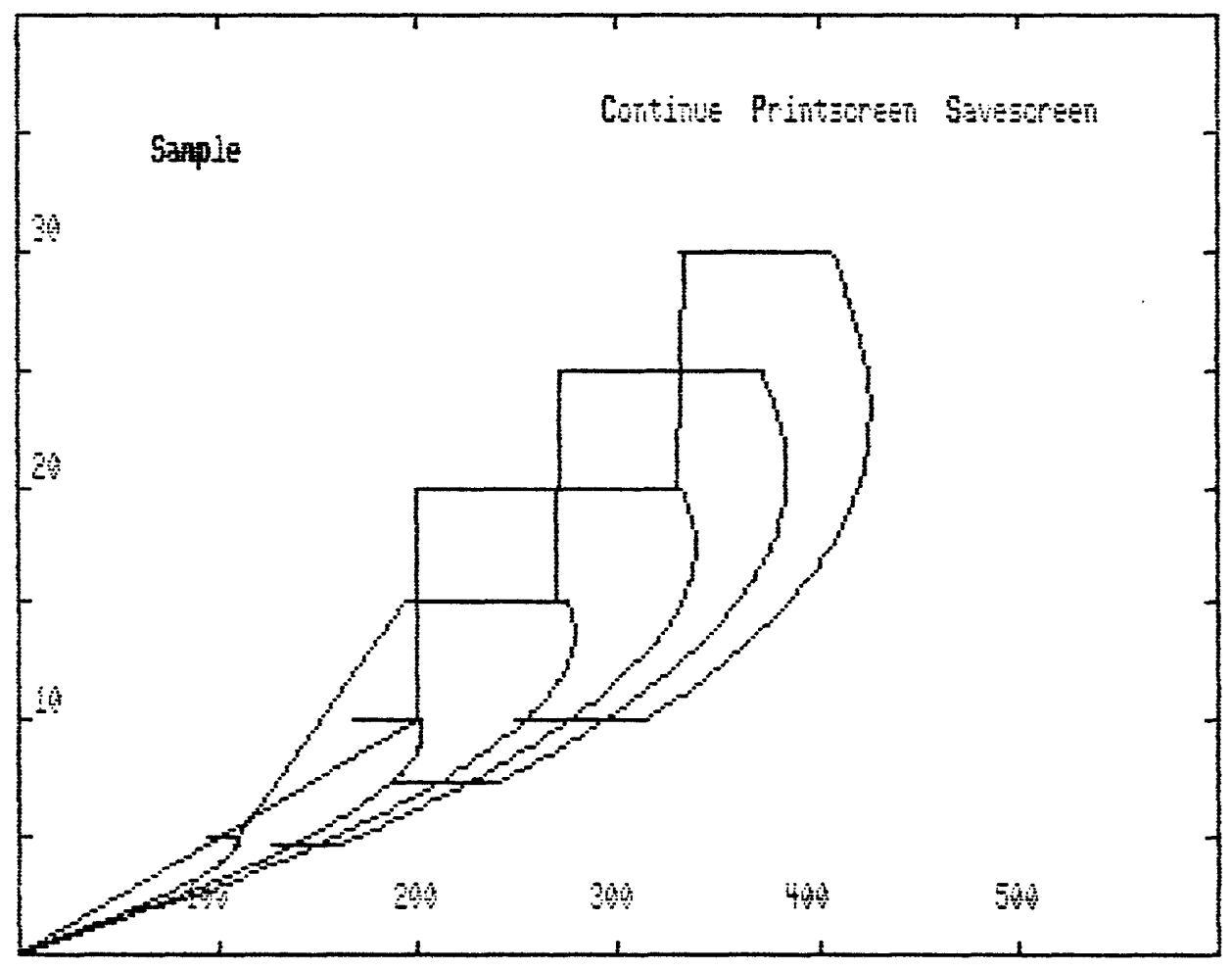

Figure 5. 


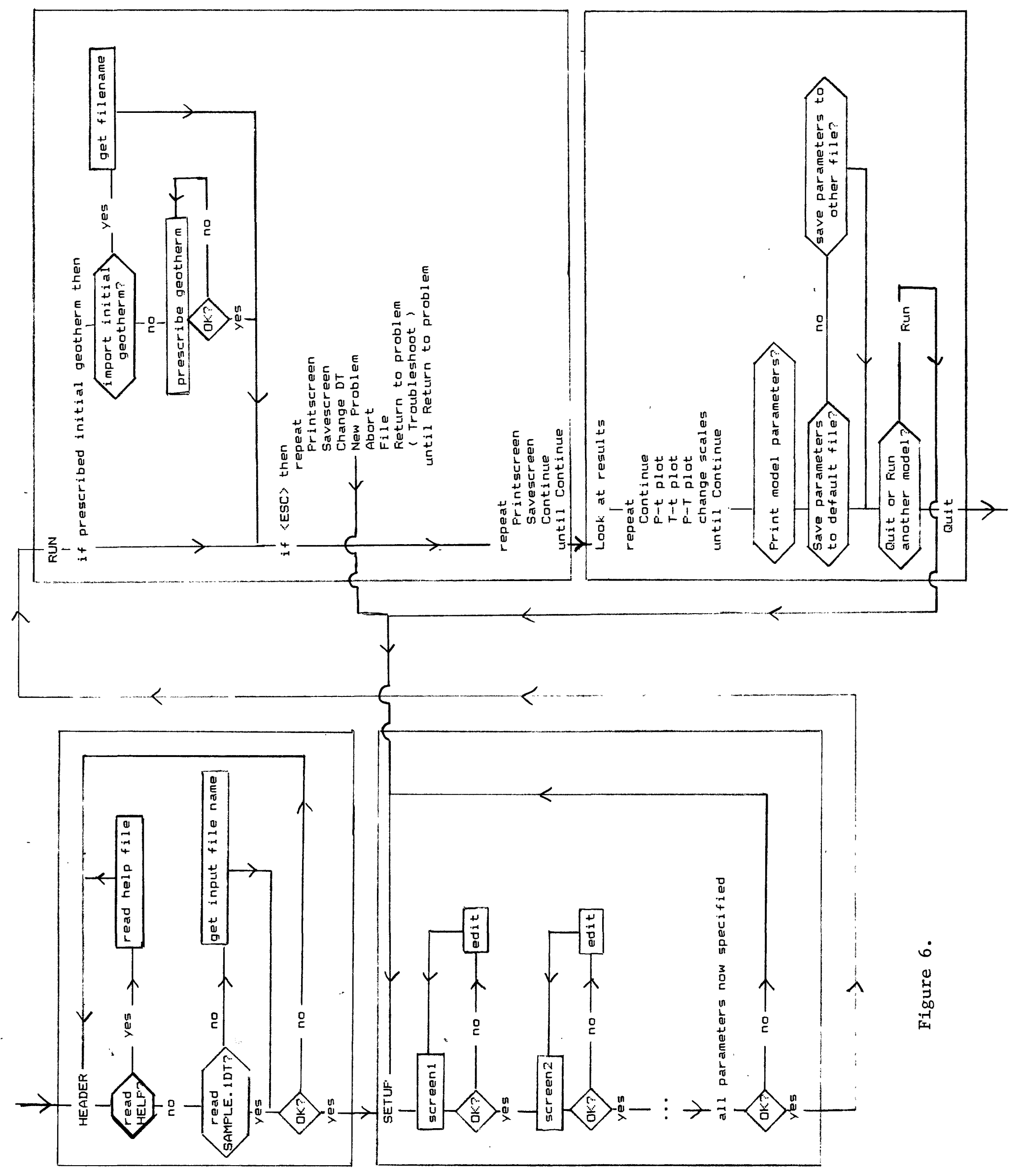

